\title{
Gradhiva
}

GRADHIV

Revue d'anthropologie et d'histoire des arts

\section{Carlo Severi et Julien Bonhomme (dir.), Paroles en actes}

Paris, L'Herne, coll. « Cahiers d'anthropologie sociale » 5, 2009, 188 p.

\section{Valentina Vapnarsky}

\section{(2) OpenEdition}

\section{Journals}

Édition électronique

URL : http://journals.openedition.org/gradhiva/2103

DOI : 10.4000/gradhiva.2103

ISSN : 1760-849X

\section{Éditeur}

Musée du quai Branly Jacques Chirac

Édition imprimée

Date de publication : 18 mai 2011

Pagination : 228-231

ISBN : 978-2-35744-042-5

ISSN : 0764-8928

\section{Référence électronique}

Valentina Vapnarsky, «Carlo Severi et Julien Bonhomme (dir.), Paroles en actes », Gradhiva [En ligne],

13 | 2011, mis en ligne le 18 mai 2011, consulté le 21 septembre 2020. URL : http://

journals.openedition.org/gradhiva/2103; DOI : https://doi.org/10.4000/gradhiva.2103

Ce document a été généré automatiquement le 21 septembre 2020.

(c) musée du quai Branly 


\section{Carlo Severi et Julien Bonhomme (dir.), Paroles en actes}

Paris, L'Herne, coll. « Cahiers d'anthropologie sociale » 5, 2009, 188 p.

Valentina Vapnarsky

\section{RÉFÉRENCE}

Carlo Severi et Julien Bonhomme (dir.), Paroles en actes. Paris, L'Herne, coll. « Cahiers d'anthropologie sociale » 5, 2009, $188 \mathrm{p}$.

1 On se réjouira de la parution récente d'une publication en français sur la pragmatique et l'anthropologie, touchant par là même à certains thèmes centraux en anthropologie linguistique. Le volume $\mathrm{n}^{\circ} 5$ des Cahiers d'anthropologie sociale nous offre sept articles de grande qualité qui développent des perspectives stimulantes sur l'interaction et les pratiques rituelles. Le titre de ce numéro joue de la notion austinienne devenue classique d' " actes de parole », pour imaginer une "pragmatique de l'anthropologie » où la communication linguistique serait mieux articulée à d'autres modes sémiotiques, et proprement intégrée aux modalités sociales de l'interaction. Partant d'une double critique adressée à l'anthropologie d'une part, pour sa prise en compte trop minimale des faits discursifs - même lorsqu'il s'agit de souligner l'efficace des paroles -, et à la pragmatique d'autre part, pour sa focalisation sur la communication parlée et en particulier la grammaire, Severi et Bonhomme introduisent ainsi l'idée originale d'une pragmatique qui serait spécifique à l'anthropologie, et peut-être même au rituel.

2 Il faut lire le premier article, celui de Severi - qui comble avec ceux de Rumsey et de Hanks notamment, une introduction au volume que d'aucuns pourront trouver trop fugace -, pour comprendre l'affirmation de l'introduction, reprise en quatrième de couverture, selon laquelle la pragmatique « se limite [...] à l'étude de tout ce qui [...] est explicitement formulé à travers des moyens appartenant à la langue » (p. 9). Difficile en effet de ne pas s'interroger sur le terme "explicitement» alors qu'un des apports fondamentaux de la pragmatique, notamment à partir des travaux de Paul Grice, est 
d'avoir montré que l'essentiel de ce qui est communiqué l'est de façon implicite, et doit donc être inféré d'après le contexte, comme le rappelle du reste Hanks dans sa contribution au volume. En outre, si la pragmatique est née de l'étude du langage, ce fut sans écarter les autres modes de communication, depuis l'idée princeps de Grice que le sens repose fondamentalement sur une reconnaissance d'intention, indépendante en principe du langage et des conventions, jusqu'à la théorie de la pertinence de Sperber et Wilson ${ }^{1}$ qui entend être un modèle cognitif général de la communication.

3 Il est toutefois vrai qu'au fil de leurs avancées, ces travaux se sont resserrés sur la langue, au point que, dans de nouvelles théories, la pragmatique devient une composante centrale de la grammaire, non plus reléguée à une phase subséquente à la sémantique mais jouant un rôle crucial dans l'interface entre les structures syntaxiques et les représentations sémantiques ${ }^{2}$. L'affirmation des éditeurs que la pragmatique relève pour les linguistes du domaine de l'explicite semble donc reposer sur le constat de l'intérêt porté aux éléments grammaticaux pris comme indices des composantes variées du contexte d'énonciation, et embrayeurs des cadres participatifs et interprétatifs des énoncés, plus que sur une définition consensuelle. Celle qu'ils reprennent pour étayer leur affirmation provient certes d'un ouvrage d'introduction à la pragmatique désormais classique : «La pragmatique est l'étude des relations entre langage et contexte qui sont grammaticalisées, ou encodées dans la structure d'une langue ${ }^{3}$ ». Il n'est pas inutile cependant de rappeler que la caractérisation citée est soumise à critique par Levinson au cours d'une longue exploration définitoire, où sont évaluées une demi-douzaine d'autres propositions. Même si le centre d'intérêt reste celui du langage, et la préoccupation majeure celle de se distinguer de la sémantique, des aspects qui intéressent particulièrement les anthropologues y sont intégrés et problématisés: relations d'interdépendance entre langage, énoncés et contexte; difficulté d'une caractérisation formelle de ce dernier ; critique de la notion de contexte approprié ; nécessité de prise en compte de la réalité des usages et des phénomènes d'exploitation des règles. Ces derniers aspects doivent ouvrir la voie à l'étude des traits émergents de l'interaction, des stratégies communicationnelles situées et de l'inventivité des locuteurs ou acteurs sociaux.

4 C'est cette voie cependant, comme le soulignent chacun à sa façon Severi, Rumsey ou Boltanski dans ce numéro, qui manque à être davantage explorée dans les travaux de pragmatique, souvent ancrés à l'excès dans les notions de convention ou de normes (normes d'usages, conventions conversationnelles, règles inférentielles, etc.), et qui a inspiré depuis un certain temps des travaux en anthropologie linguistique. Aux notions fondamentales de sous-détermination sémantique, d'intentionnalité et d'inférence communicationnelle issues de Grice, et à la performativité et l'efficacité d'Austin, sont articulés plusieurs apports : ceux de la sémiotique holiste de Peirce et de l'indexicalité (Jakobson, Silverstein), et ceux d'une conception dynamique et fondamentalement interactionnelle des " jeux de langages » - reprenant l'expression de Wittgenstein - qui donne forme, sens et fonction aux énoncés employés, tout en les alliant aux autres modalités de communication et d'action sociale. Des travaux plus récents intègrent ces approches à celles de la cognition et de la psychologie dans le cadre plus ambitieux d'un champ de recherche interdisciplinaire sur la compréhension de la socialité humaine ${ }^{4}$. $\mathrm{Au}$ cœur de ces travaux, comme des articles proposés dans Paroles en actes, figurent d'une part l'analyse des interactions sociales et de l'articulation fine et complexe qui s'y tisse entre les modalités linguistiques et non linguistiques de communication et d'action; et, d'autre part, une réflexion sur la constitution et la recomposition 
constante de cet ensemble de savoirs, de principes, d'orientations et de terrains communs, partagés ou à partager, à la fois point de départ et point de mire des interactions communicationnelles. Une des originalités du volume est l'accent mis sur les contextes rituels et la dynamique qui s'y exerce entre des cadres d'interactions multiples et éventuellement contradictoires. En ce sens, les éditeurs s'inscrivent également, en même temps qu'ils s'en démarquent, dans le prolongement d'une réflexion qui unissait déjà anthropologie et pragmatique ${ }^{5}$, et qui, partant de l'interaction dialogique, explorait plutôt les relations d'affinité entre les domaines du quotidien et du rituel.

5 Les articles du Cahier se concentrent ainsi pour la plupart sur les situations rituelles et la dynamique (superposition, duplication, intrication, fusion...) des cadres d'interaction et de participation qui les caractérisent. On peut voir dans cette approche un élargissement des analyses sur les processus d'enchâssement et de transpositions de cadres telles que celles de $\mathrm{Hanks}^{6}$ et Haviland ${ }^{7}$. Pour certains des auteurs, c'est plus particulièrement l'occasion de développer l'hypothèse proposée par Houseman et Severi ${ }^{8}$ selon laquelle les rituels se définissent par la condensation d'identités plurielles ou contradictoires. Le lecteur trouvera un très riche ensemble d'analyses quant à la spécificité du rituel par rapport à l'échange ordinaire, déclinées dans l'étude des statues funéraires grecques, formes d'icônes parlantes, de Severi, des rituels d'échanges intergroupes ku waru (Nouvelle-Guinée) de Rumsey, des modes de transmission des registres relationnelschamaniques sharanahua (Amazonie) de Déléage, des rituels de divination mayas de Hanks, de la rumeur du vol de sexe en Afrique de Bonhomme, ou des dispositifs de résolution de conflits (ho'oponopono à Hawaï, becharaa' des Semai, Malaisie, gruarutha à Santa Isabel) de Berthomé. L'article final de Boltanski, tout en présentant la perspective d'une sociologie qui a su inscrire une approche pragmatique dans sa réflexion depuis une vingtaine d'années, témoigne combien l'importance accordée à l'action, ainsi qu'aux capacités critiques et plus largement réflexives des acteurs, renouvelle la façon de concevoir les institutions, depuis les cérémonies jusqu'aux instances d'évaluation et de certification de « ce qui est ».

6 Alors que Severi soutient l'hypothèse d'une complexité spécifique de l'action ri-tuelle, laquelle procèderait de phénomènes de contextualisation qui lui seraient propres, et que Déléage s'inscrit en ce sens lorsqu'il considère qu'un certain type d'articulation de cadres d'interaction (nommément la duplication) définit le caractère rituel de la transmission chamanique, d'autres auteurs adoptent une position moins tranchée. Ainsi, si Hanks insiste sur l'extrême asymétrie qui caractérise les perspectives des participants du rituel analysé, il souligne par ailleurs que les stratégies communicatives employées par le chamane sont celles du discours ordinaire, et que ses "procédés sémiotiques [...] sont tout à fait banals même s'il les maîtrise à la perfection » (p. 110). Rumsey, quant à lui, montre que certains énoncés informels, tels que ceux qu'il appelle "ventriloques », fréquemment utilisés dans les discours adressés aux enfants chez les Kaluli et les Ku Waru (où les parents parlent en adoptant le champ indexical de l'enfant), partagent différents traits des interactions rituelles, en particulier la superposition des cadres d'interaction. Au terme de la lecture de l'ouvrage, on rejoindra donc Rumsey pour considérer que la question épineuse de la relation entre interactions rituelles et ordinaires reste ouverte, et d'autant plus passionnante.

7 Des hypothèses variées transparaissent également concernant le moteur de la dynamique des cadres participatifs, qui sous-tend l'interprétation par les acteurs eux- 
mêmes du dit et du vécu. Cette dynamique repose-t-elle principalement sur des jeux d'attributions d'identités entre les entités en présence, indépendants des échanges verbaux ? Ou repose-t-elle plutôt à la fois sur des jeux grammaticaux, tels que ceux des embrayeurs déictiques, épistémiques, ou temporels qui semblent pouvoir transformer en un clin de morphème la configuration de la situation d'énonciation, de l'horizon et l'action du discours? Par exemple, on observera comment le passage au fil d'une phrase du parfait au passé reculé ramène une identité segmentaire à une implication individuelle dans les "discours de combat» ku waru; comment l'abandon d'un marqueur de discours rapporté au profit d'un morphème épistémique d'expérience directe scelle une relation d'apprentissage et parachève l'identification performative du novice au chamane et aux esprits chez les Sharanahua ;commentl'usage contrasté de déictiques spatiaux compose des cadres d'inter-

actions disjoints, asymétriques et éventuellement concomitants, à des moments clés des rituels de divination mayas.Mais la dynamique ne repose-t-elle pas également sur l'orientation corporelle des participants, incluant gestes et regards, qui dessine des champs d'adresse, d'inclusion et d'exclusion, ou encore des centres et des périphéries ? On suit minutieusement ces effets dans la description des mouvements du chamane maya par Hanks, dans les dispositions spatiales changeantes des participants aux séances de résolution de conflit comparées par Berthomé, ou encore dans la façon dont une simple situation de coprésence peut être perçue comme une effraction violente de l'intimité et subitement se transmuer en une relation asymétrique dangereuse, voire fatale, dans le cas des accusations de vol de sexe analysées par Bonhomme.

Dans tous les cas, l'ensemble des articles de Paroles en actes souligne l'importance des canaux ou modes de communication non linguistiques et scrute la façon dont ils remplacent et supplantent les discours, ou plus souvent s'y combinent. Des réflexions sont menées sur les liens entre la communication verbale, la gestuelle et le jeu des regards, l'orientation corporelle et les dispositions spatiales, les modes de signification iconique - c'est-à-dire la représentation imagée -, la contribution des états émotionnels, individuels ou collectifs, enrichissant les travaux sur la multimodalité. Dans cette exploration anthropologique qui revient sur les sémiotiques de Pierce et de Bühler, on lira notamment l'analyse de Severi sur l'articulation entre les modes de représentation iconique et indexical, et leurs implications réciproques dans la communication. Les hypothèses sont étayées par une étude captivante sur les identités enchâssées (via la parole qui leur est prêtée) des statues funéraires de la Grèce ancienne, qui inspirera vivement la réflexion sur les relations entre représentation imagée et texte/parole, leurs liens au registre rituel, ainsi que sur les processus d'attribution de subjectivité aux artefacts. On retiendra aussi le passage de l'article de Hanks concernant les images perçues par le chamane maya dans ses cristaux divinatoires, traitées comme des signes tangibles d'intentions communicatives, et intégrées en propre avec leur support cristallin aux cadres d'interactions de l'activité rituelle thérapeutique.

Un autre thème crucial abordé par plusieurs des articles est celui de la construction d'un savoir partagé ou commun, et plus fondamentalement d'une orientation mutuelle ou d'un terrain d'entente, pour reprendre la notion de Clark (joint commitment) qui est au centre de l'article de Hanks. Au-delà d'une conception dynamique du contexte, et de l'idée que les échanges verbaux impliquent de façon inhérente des transformations de la perspective et des savoirs mutuels des participants ${ }^{9}$, il s'agit de comprendre comment les interactants construisent un champ d'interprétation commun, qu'il soit 
collaboratif ou antagonique, et peuvent éventuellement converger vers une issue acceptée de tous, voire efficace. Les cas étudiés sont d'autant plus significatifs qu'ils présentent, pour la plupart, des interactions marquées au départ par une forte asymétrie de perspective et de savoir. On est là au cœur de l'analyse des processus de conviction, souvent évanescents, parfois sédimentés, et de celui des constitutions de croyances, de validation et de cristallisation des représentations.

De façon tout à fait intéressante, plusieurs articles soulignent l'importance de l'incertitude ou de l'incomplétude des représentations attachées à certaines situations et à certains énoncés. Boltanski part même d'une " position originelle [...] dans laquelle règne une incertitude radicale» (p.164-165), et d'une tension constitutive entre des moments pratiques et des dispositifs métapragmatiques de confirmation qui éloignent cette incertitude, alors que la réflexivité critique la fait sans cesse resurgir. Incertitude et incomplétude ne sont donc pas conçues comme représentant nécessairement des manques à combler, à la différence des principes classiques de la pragmatique de reconstruction du sens, mais apparaissent au contraire comme une composante essentielle de certaines formes d'efficacité ou de coordination et de contrainte sociale. Alors que dans les dispositifs de conciliation comparés par Berthomé, l'établissement d'un savoir commun contribue à réduire l'incertitude que les dissensions font peser sur les relations, et à affermir ces dernières, les rituels chamaniques analysés par Déléage et Hanks, ainsi que les situations de contact entre inconnus qui suscitent l'accusation violente de vol de sexe et sa rumeur étudiées par Bonhomme, montrent le rôle crucial du sentiment d'incertitude et de délégation de savoir et de contrôle - qui lient l'épistémique à l'émotionnel -, dans la transformation de l'expérience du novice ou du patient, de l'accusateur ou de la victime, et dans la dynamique efficace de l'action.

11 Pour toutes les réflexions que ces différents thèmes inspirent, et bien d'autres que les analyses spécifiques de chaque article nourrissent et que nous laissons au lecteur le plaisir de découvrir, on ne peut que recommander la lecture de cet ouvrage. En espérant que ceci contribue à développer des recherches alliant chaque fois plus finement les différentes modalités d'action et de communication, de construction et d'interprétation du sens, champ commun de disciplines qui semblent avoir plus à gagner de la complémentarité de leurs points de vue et de leur expertise que de la création de nouvelles frontières.

\section{NOTES}

1. La Pertinence. Paris, Minuit, 1989 [1986].

2. Stephen C. Levinson, Presumptive Meanings. The Theory of Generalized Conversational Implicature. Cambridge (Mass.), MIT Press, 2000.

3. "Pragmatics is the study of those relations between language and context that are grammaticalized, or encoded in the structure of a language. " (Stephen C. Levinson, Pragmatics. Cambridge, Cambridge University Press, 1983 : 9).

4. Nick J. Enfield et Stephen C. Levinson (éd.), Roots of Human Sociality. Culture, cognition and Interaction. Oxford, Berg, 2006. 
5. Aurore Monod Becquelin et Philippe Erikson (éd.), Les Rituels du dialogue. Nanterre, Société d'ethnologie, 2000.

6. Referential Practice: Language and lived space among the Maya. Chicago, University of Chicago Press, 1990.

7. « Warding of witches: Voicing and dialogue in Zinacantec prayer », in Aurore Monod Becquelin et Philippe Erikson (éd.), op. cit., 2000 : 367-400.

8. Naven ou le donner à voir. Essai d'interprétation de l'action rituelle, Paris, CNRS Éditions-Éditions de la MSH, 1994.

9. Voir Alessandro Duranti et Charles Goodwin, Rethinking Context: Language as an Interactive Phenomenon. Cambridge, Cambridge University Press, 1992.

\section{AUTEURS}

\section{VALENTINA VAPNARSKY}

vapnarsk@vjf.cnrs.fr 